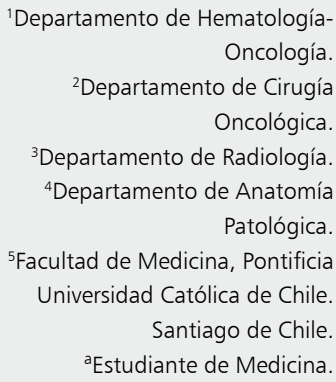

'Departamento de HematologíaOncología. ${ }^{2}$ Departamento de Cirugía Oncológica.

${ }^{3}$ Departamento de Radiología.

${ }^{4}$ Departamento de Anatomía Patológica.

${ }^{5}$ Facultad de Medicina, Pontificia Universidad Católica de Chile. Santiago de Chile.

aEstudiante de Medicina.

Los autores declararon no tener conflictos de intereses.

Recibido el 23 de septiembre de 2013, aceptado el 10 de abril de 2014

Correspondencia a:

Dr. César Sánchez R. Departamento HematologíaOncología, Centro de Cáncer. Pontificia Universidad Católica de Chile.

Diagonal Paraguay 319, Santiago, Chile.

Teléfono: 56-2- 23546919 csanchez@med.puc.cl

\section{Cáncer de mama metastásico. Caracterización de una cohorte según subtipos}

\author{
CÉSAR SÁNCHEZ R. ${ }^{1}$, FRANCISCO ACEVEDO C. ${ }^{1,5}$, MILITZA PETRIC G. ${ }^{2,5}$, \\ HÉCTOR GALINDO A. ${ }^{1,5}$, FRANCISCO DOMÍNGUEZ C. ${ }^{2,5}$, \\ AUGUSTO LEÓN R., ${ }^{2,5}$, DRAVNA RAZMILIC V., \\ CAROLINA CEBALLOS. ${ }^{5, \mathrm{a}}$, FERNANDO ESPINOZA ${ }^{5, \mathrm{a}}$, \\ M. ELENA NAVARRO O., ${ }^{3,5}$, DAVID ODDÓ B. ${ }^{4,5}$, MAURICIO CAMUS A.,
}

\section{Survival of patients with metastatic breast cancer according to pathological types of tumors}

Background: The prognosis of breast cancer $(B C)$ is in part determined by the stage at diagnosis and its pathological characteristics. Aim: To evaluate the association between survival of women with metastatic breast cancer and pathological features of the tumor. Patients and Methods: We obtained clinical and pathological data from patients diagnosed with a metastatic $B C$ between 1999 and 2013. The expression of estrogen (ER) and progesterone (PR) receptors and human epidermal growth factor receptor 2 (HER2) was determined by immunohistochemistry. Clinicopathological subtypes were defined as: Luminal A: ER or PR positive, HER2 negative, histological grade (HG) 1 or 2; Luminal B: $E R$ or $P R$ positive, HER2 negative or positive or $H G 3$; triple negative (TN): $E R$, $P R$ and HER2 negative, independent of the $H G$, positive HER2: ER, $P R$ negative and HER2 positive, independent of HG. We analyzed survival based on these subtypes. Results: We identified 54 patients aged 24 to 85 years, with metastatic $B C$ at diagnosis. Seventy five percent had luminal tumors; $19.6 \%$ HER2 positive and $7.8 \%$ were TN. In $61 \%$ of evaluable tumors, HG was classified as 3. The frequency of HER2 positive and high HG tumors was greater in these patients with metastatic $B C$ than in a non-metastatic local $B C$ cohort. Survival was higher among patients with Luminal tumors than in women with non-Luminal cancer (56.4 and 11.4 months, respectively, $p=0.04$ ). Conclusions: Patients with metastatic $B C$ at diagnosis often had HER2 positive tumors and high HG. As in other studies, ER positive tumors had a better survival.

(Rev Med Chile 2014; 142: 428-435)

Key words: Breast Neoplasms; Classification; Neoplasm metastasis; Prognosis. 
plasia en diferentes subtipos de acuerdo a la evaluación de genes relacionados, principalmente a la expresión del RE, RP, HER2 y de proliferación ${ }^{5}$. Sin embargo, la determinación de estos perfiles moleculares, basados en estudios de la expresión génica, es de alto costo y no está disponible en la práctica clínica habitual.

Esta clasificación molecular tiene un correlato en la anatomía patológica convencional, logrando predecir el pronóstico y sugerir respuestas a tratamiento, especialmente para tumores localizados, no metastásicos. Tumores con perfil molecular Luminal A corresponden generalmente a aquellos con alta expresión de RE y/o RP, HER2 negativos y con bajo índice de proliferación; este último medido a través de un marcador inmuno-histoquímico (IHC) llamado Ki67. Tumores Luminales B, por otro lado, presentan menor expresión de RE/RP, pueden ser positivos para HER2 y expresan altos niveles de Ki67. Neoplasias basales en la clasificación molecular corresponden, en su mayoría, aunque no exclusivamente, a tumores triple negativos $(\mathrm{TN})^{5,6}$.

Alternativas basadas en parámetros IHC clásicos se han comparado con los clasificadores genéticos, logrando una buena correlación en la predicción del pronóstico y en el patrón de recaídas de pacientes con CM en etapas precoces ${ }^{6}$.

Nuestro objetivo es caracterizar clínica y patológicamente a pacientes con cáncer de mama metastático (CMM) al debut y evaluar su sobrevida según sub tipos clínico-patológicos, definidos de acuerdo a patrones IHC en la biopsia.

\section{Pacientes y Método}

Se realizó un estudio descriptivo en el cual se analizaron las historias clínicas de pacientes con diagnóstico de $\mathrm{CM}$, tratadas entre enero del año 1999 y mayo de 2013 en el Centro de Cáncer de la Pontificia Universidad Católica de Chile, Santiago, Chile. Registramos: edad, hallazgos patológicos del tumor, tipo de tratamiento sistémico (terapia endocrina y/o quimioterapia), sobrevida global (SG) y especifica por la enfermedad (SEE). La SG fue definida como el tiempo transcurrido desde la biopsia diagnóstica inicial hasta el fallecimiento por cualquier causa. La fecha y causas de muerte se obtuvieron de datos de los certificados de defunción emitidos por el Registro Civil y de registros clínicos. Para la SEE se definió el mismo período que la SG y se contabilizaron sólo las pacientes que fallecieron de CM.

El análisis patológico incluyó: tipo histológico, grado de diferenciación según la clasificación de Elston y Bloom, modificada por Elston y Ellis ${ }^{7}$, estudio de RE y RP, en escala de $0-100 \%$, HER 2 escala de $+\mathrm{a}+++$. Todos por métodos de IHC. Siendo para HER2: + negativo, ++ incierto evaluado adicionalmente por inmunofluorescencia in situ (FISH) y +++ positivo. Se definió el subtipo tumoral clínico-patológico según los siguientes elementos: Luminal A (RE + y/o RP +, GH 1-2, HER2 negativo); Luminal B ( $\mathrm{RE}+\mathrm{y} / \mathrm{o} \mathrm{RP}+, \mathrm{GH}$ 3 y /o HER 2 positivo), TN (RE -, RP -, y HER negativo), HER2+ (RE - y RP -, HER 2 positivo) $(\text { Tabla } 1)^{8}$. Durante el período del estudio no se contó con Ki67, por lo que usamos el GH como un parámetro asociado al valor de $\mathrm{Ki} 67^{9}$.

Comparamos las características clínicas y patológicas de la cohorte de pacientes identificadas con CMM y de aquellas con CM no metastásico. Analizamos los datos con programa SPSS versión 21.0, utilizando estadística descriptiva. Calculamos la sobrevida según Kaplan-Meier y se compararon las curvas con test de Log-Rank. Consideramos diferencias estadísticamente significativas con valores de $\mathrm{p} \leq 0,05$.

\section{Resultados}

En el período de estudio, identificamos 54 pacientes con CMM al diagnóstico, 3,6\% de las pacientes tratadas en este centro. Sus características

\section{Tabla 1. Definición de subtipo clínico y patológico}

\begin{tabular}{|c|c|}
\hline $\begin{array}{l}\text { Subtipo } \\
\text { molecular }\end{array}$ & Expresión IHQ \\
\hline Luminal A & $\mathrm{RE}+; \mathrm{PR}+$; HER2-;Ki67 < 14\%* \\
\hline Luminal B & $\mathrm{RE}+; \mathrm{PR} \pm ; \mathrm{HER} 2 \pm ; \mathrm{KI} 67>14 \%$ * \\
\hline HER2 + & RE -; PR-; HER2 + \\
\hline Triple negativo & RE -; PR-; HER2- \\
\hline
\end{tabular}

*En ausencia de Ki67, se ha utilizado el grado histológico (GH) para diferenciar Luminal A (GH 1-2) y Luminal B (GH3). IHC: Inmuno-histoquímica; RE: receptor de estrógenos; RP: receptor de progesterona; HER2: receptor del factor de crecimiento epidérmico tipo 2 . 
se muestran en la Tabla 2. Todas eran mujeres. La mediana de edad fue de 57 años (margen 24 a 85). En 3 pacientes no tuvimos información suficiente de la biopsia para tipificar el tumor. El 72,5\% (37/51) tenía tumores $\mathrm{RE}+$ (Luminal $\mathrm{A}=10, \mathrm{Lu}-$ minal $\mathrm{B}=18$, Luminal no especificado=9), HER2 $+10 / 51(19,6 \%)$ y $4 / 51(7,8 \%)$ pacientes eran TN. Al comparar estos hallazgos con nuestras pacientes con CM no metastásico observamos diferencias estadísticamente significativas en la expresión de HER2 y de tumores clasificados como Luminal A. La prevalencia de tumores HER2 positivo (Luminal B/HER2+ y HER2+) fue significativamente mayor en las pacientes con CMM que en nuestra cohorte local de CM no metastásico (37,2\% vs $12,3 \%$; p < 0,0001). El $18,5 \%$ de los tumores en mujeres con CMM correspondía al subtipo HER2+ versus 5,1\% en la población con CM localizado ( $\mathrm{p}=0,0001)$ (Tabla 3). Además, la presencia de tumores poco diferenciados ( $\mathrm{GH} 3$ ) fue mayor en CMM al debut: 60,9\% de tumores GH 3 en pacientes con CMM y $43,4 \%$ GH 3 en pacientes con CM no metastásico $(\mathrm{p}=0,03)$ (Figura 1 y Tabla 2). Con una mediana de seguimiento del grupo con CMM de 26,7 meses, obtuvimos una mediana de SG y SEE de 39,8 y 56,4 meses, respectivamente (Figuras 2 y 3 ). La SEE en tumores con GH 1-2 fue de 43 meses versus 19,5 meses en tumores con GH 3 ( $\mathrm{p}=0,47)$. Al comparar la SG y SEE según subtipos histológicos, se observó que la mediana de sobrevida de los tumores Luminales fue de 56,4 meses y la de los no Luminales 11,4

Tabla 2. Características clínicas y patológicas de 54 pacientes con cáncer de mama metastásico a la presentación de la enfermedad y de 1.492 pacientes con cáncer de mama no metastásico

\begin{tabular}{|c|c|c|c|c|c|}
\hline & \multicolumn{2}{|c|}{$\begin{array}{c}\text { CMM al debut } \\
\text { n: } 54^{\# \#}\end{array}$} & \multicolumn{2}{|c|}{$\begin{array}{c}\text { CM no metastásico } \\
\text { n: } 1.492^{\# \# \#}\end{array}$} & $\mathbf{p}$ \\
\hline Edad (años; margen) & \multicolumn{2}{|c|}{$57(24-85)$} & \multicolumn{2}{|c|}{$55(25-95)$} & 0,2 \\
\hline RE/RP positivos & $37 / 51$ & $72,5 \%$ & 1.128 & $81,6 \%$ & 0,09 \\
\hline RE/RP negativos & $14 / 51$ & $27,4 \%$ & 253 & $18,3 \%$ & \\
\hline HER2 positivo* & $19 / 51$ & $37,2 \%$ & 184 & $12,3 \%$ & $<0,0001^{\#}$ \\
\hline $\mathrm{GH} 1$ y 2 & $16 / 41$ & $39 \%$ & 661 & $56,6 \%$ & $0,03^{\#}$ \\
\hline $\mathrm{GH} 3$ & $25 / 41$ & $60,9 \%$ & 507 & $43,4 \%$ & \\
\hline
\end{tabular}

*HER 2 positivo independiente del estado de RE. "Diferencia estadísticamente significativa. \#\#3 pacientes sin información de inmuno-histoquímica y 13 pacientes sin información de GH. \#\#111 pacientes sin información de inmuno-histoquímica y 324 pacientes sin información de GH. CM: Cáncer de mama; CMM: Cáncer de mama metastásico; RE: receptor de estrógeno; RP: receptor de progesterona; HER2: Receptor del factor de crecimiento epidérmico tipo 2; GH: grado histológico.

Tabla 3. Subtipos clínico-patológicos de 54 pacientes con cáncer de mama metastásico a la presentación de la enfermedad y 1.492 pacientes con cáncer de mama no metastásico

\begin{tabular}{|lccccc|}
\hline Subtipos & \multicolumn{2}{c}{$\begin{array}{c}\text { CMM al debut } \\
\text { n: } \mathbf{5 4}^{\# \#}\end{array}$} & \multicolumn{2}{c}{$\begin{array}{c}\text { CM no metastásico } \\
\text { n: 1.492 }\end{array}$} & p \\
Triple negativo & $4 / 51$ & $7,8 \%$ & $160 / 1.361$ & $11,7 \%$ & 0,4 \\
Luminal A & $10 / 51$ & $19,6 \%$ & 569 & $41,8 \%$ & $0,004^{\#}$ \\
Luminal B & $18 / 51$ & $35,2 \%$ & 399 & $29,3 \%$ & 0,27 \\
Luminal NE & $9 / 51$ & $17,6 \%$ & 163 & $11,9 \%$ & - \\
HER2** & $10 / 51$ & $18, \%$ & 70 & $5,1 \%$ & $<0,0001^{\#}$ \\
\hline
\end{tabular}

**HER positivos, con RE/RP negativos. "Diferencia estadísticamente significativa. ${ }^{* \# 3 ~ p a c i e n t e s ~ s i n ~ i n f o r m a c i o ́ n ~ q u e ~ n o s ~ p e r m i t a ~}$

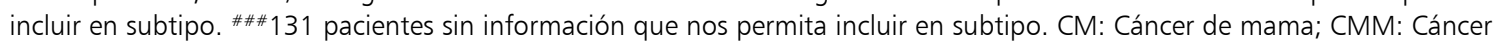
de mama metastásico; RE: receptor de estrógenos; RP: receptor de progesterona; HER2: Receptor del factor de crecimiento epidérmico tipo 2 . 

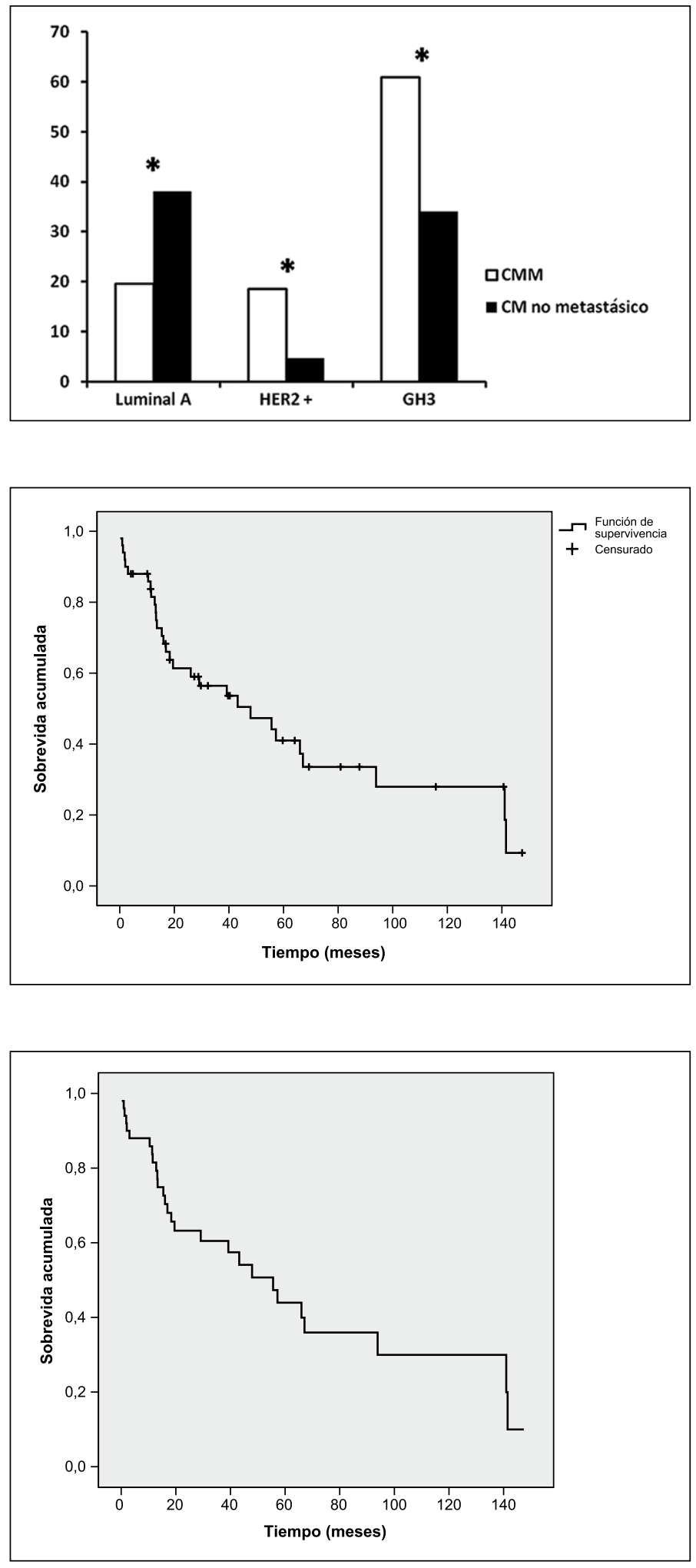

Figura 1. Características clínico-patológicas con diferencias estadísticamente significativas entre cáncer de mama metastásico (CMM) y cáncer de mama (CM) no metastásico. *Diferencia estadísticamente significativa entre grupos. GH3: Grado histológico 3.

Figura 2. Sobrevida global de una cohorte de pacientes con cáncer de mama metastásico al debut, tratados en el Centro de Cáncer, Pontificia Universidad Católica de Chile, entre los años 1999 y 2013.

Figura 3. Sobrevida específica por enfermedad de una cohorte de pacientes con cáncer de mama metastásico al debut, tratados en el Centro de Cáncer, Pontificia Universidad Católica de Chile, entre los años 1999 y 2013. 


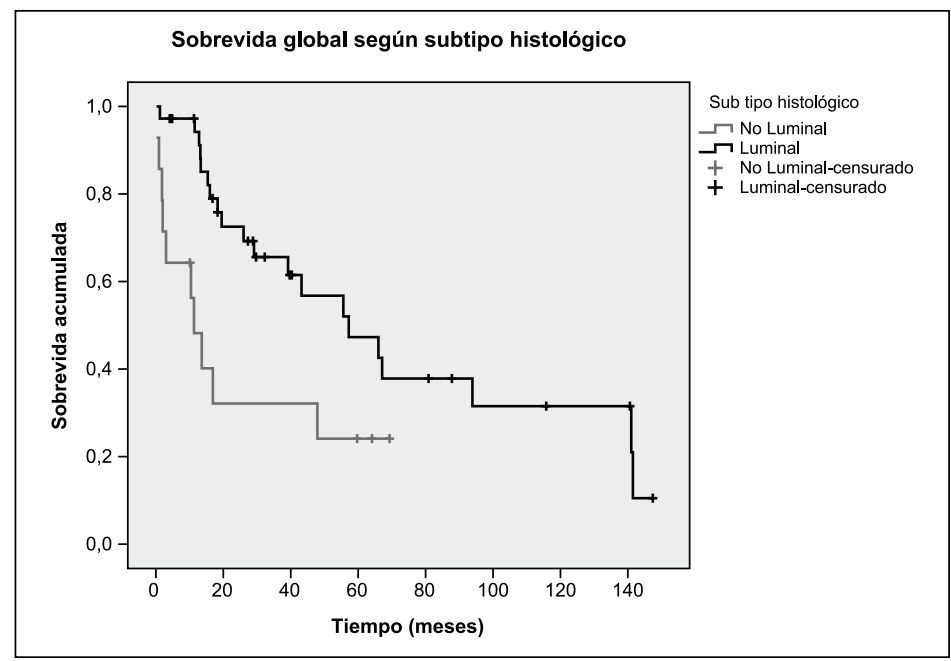

Figura 4. Sobrevida global de una cohorte de pacientes con cáncer de mama metastásico al debut, tratados en el Centro de Cáncer, Pontificia Universidad Católica de Chile, entre los años 1999 y 2013, según subtipo clínicopatológico.

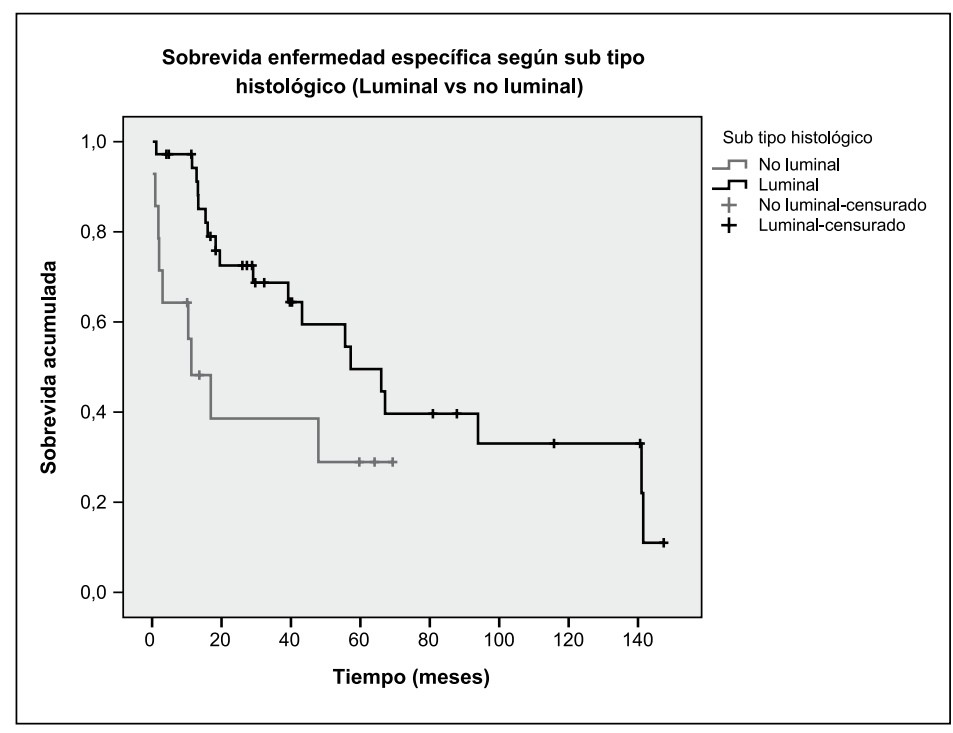

Figura 5. Sobrevida específica por enfermedad de una cohorte de pacientes con cáncer de mama metastásico al debut, tratados en el Centro de Cáncer, Pontificia Universidad Católica de Chile entre los años 1999 y 2013 , según subtipo clínico-patológico. meses $(\mathrm{p}=0,04)$ (Figuras 4 y 5). Al comparar la SEE en tumores definidos como Luminal A versus Luminal B no encontramos diferencia estadísticamente significativa (55,4 meses vs 32,3 meses; respectivamente; $\mathrm{p}=0,29$ ).

\section{Discusión}

El CM se presenta en la mayoría de los casos como una enfermedad localizada, sin embargo, hasta $30 \%$ de las pacientes pueden tener una recidiva de la enfermedad ${ }^{10-12}$. La presencia de enfermedad metastásica al debut, es un evento poco frecuente ${ }^{10}$. En nuestro Centro de Cáncer correspondió a menos de 4\% del total de tumores invasores, un número similar a lo reportado en otras series ${ }^{10,11}$.

El estudio del perfil de expresión génica en $\mathrm{CM}$ evidencia la existencia de una enfermedad heterogénea. Perou y cols. fueron los primeros en describir cuatro clases moleculares de CM que ellos llamaron subtipos intrínsecos ${ }^{5}$. Aunque estos subgrupos fueron originalmente diseñados para evaluar el pronóstico del CM, pueden predecir también la respuesta a los tratamientos oncoló- 
gicos. Durante los últimos años, varias firmas de perfiles genéticos se han desarrollado y validado en estudios clínicos; sin embargo, sólo algunas de ellas están disponibles comercialmente y son factibles de usar, pues requieren tejido fijado en parafina y no tejido fresco, este último, de difícil disponibilidad en la práctica habitual ${ }^{12-14}$. Uno de los más utilizados es el Oncotype DX que calcula un puntaje de recurrencia basado en la expresión de 16 genes relacionados a $\mathrm{CM}$ en pacientes con tumores $\mathrm{RE}+$. Estos genes se relacionan a la expresión de RE, RP, HER2 y proliferación celular. El puntaje es agrupado en tres categorías: bajo, intermedio o alto $^{12}$. Si bien este test ha sido ampliamente usado en algunos lugares del mundo en la toma de decisiones en pacientes con tumores RE+ no metastásicos, existe evidencia que variables clínicas y patológicas más clásicas proveen información del pronóstico independiente del puntaje ${ }^{6}$.

Otro test genético, también validado en muestras de tejido fijado en parafina, es el Mamma Print. Dicho test utiliza información de 70 genes relacionados con proliferación, invasión, metástasis, estroma y angiogénesis. A diferencia de Oncotype DX este perfil clasifica los tumores en dos grupos: alto y bajo, sin una categoría de riesgo intermedio; y recientemente ha demostrado, en un estudio prospectivo, adicionar información a parámetros clínico-patológicos y buena reproducibilidad al ser utilizado en tejido fijado ${ }^{15,16}$.

Limitaciones técnicas y económicas impiden realizar, en la práctica clínica habitual en nuestro medio, perfiles genéticos en pacientes con CM. Por esta razón, se intenta correlacionar los subtipos intrínsecos de $\mathrm{CM}$, definidos por estos perfiles genéticos, con subtipos clínico-patológicos, definidos por clasificaciones y técnicas IHC convencionales $^{6,17}$. Este último método ha logrado una buena concordancia con los perfiles genéticos. Es así como tumores denominados "Luminales", se caracterizan fenotípicamente por expresar el RE, los tipos llamados "HER2" muestran sobreexpresión de HER2 sin expresar RE; y aquellos considerados como "Basaloides", por estudios de expresión génica, son negativos tanto para RE, RP y HER2, por lo que son conocidos como "triple

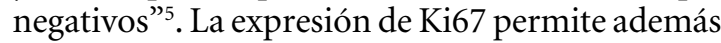
subdividir el tipo Luminal en A y B (Tabla 1). El Ki67 es una proteína nuclear no histona, expresada por células proliferantes en fases G1 tardía, $S$ y G2/M del ciclo celular; refleja por tanto, la proporción de células en proliferación y ha sido usado como un factor predictivo de respuesta a terapia, en particular a terapia endocrina $y$, en estudios recientes, a quimioterapia ${ }^{18}$.

El estudio de Ki67 no se realiza de rutina en nuestras biopsias de CM infiltrante. Su costo, la falta de estandarización en su medición (dadas variables analíticas y pre-analíticas) ${ }^{9}$ limitan su uso y actualmente no son parte del informe anatomopatológico habitual en nuestra institución. Un reciente estudio comparó el valor de tres modelos, el Oncotype DX, el PAM50 (una clasificación de subtipos basado en 50 genes) y el uso de 4 marcadores IHC (ER, PR, Ki67, HER2) para predecir el riesgo de recurrencia en tumores localizados luego de adyuvancia con terapia endocrina. Este estudio mostró un rendimiento estadísticamente similar para todos ellos ${ }^{6}$.

Parámetros clásicos, informados de rutina en la biopsia de mama, como el GH y el índice mitótico (IM) han mostrado buena correlación con la proliferación celular ${ }^{18}$. Uno de ellos es parte del índice pronóstico de Nottingham (Nottingham Prognostic Index: NPI $)^{20}$. El GH, y también el IM, están correlacionados al Ki679 .

Recientemente se ha descrito que la expresión del RP puede separar tumores Luminales A y B. Una expresión del RP mayor a $20 \%$ sería característica de tumores Luminales $\mathrm{A}^{17}$. Dado que no usamos rutinariamente este parámetro en nuestra práctica al momento de colectar los datos, hemos decidido reportar nuestros datos sin este parámetro.

La mayoría de los estudios que analizan el comportamiento clínico de los subtipos de CM están referidos a pacientes con enfermedad no metastásica, y son escasos los reportes de su valor en pacientes con CMM inicial ${ }^{21}$. El uso de estos bio-marcadores clásicos ya estandarizados, para definir "subtipos moleculares", aunque no perfecto, permite su aplicabilidad y utilidad en todo el mundo y en cualquier laboratorio de patología sin aumentar los costos y tiempo del procesamiento habitual de la muestra.

El objetivo de este estudio fue caracterizar clínica y patológicamente nuestras pacientes con CMM al debut y evaluar su sobrevida según sub tipos histopatológicos, definidos de acuerdo a patrones IHC en la biopsia, sin el uso de Ki67 $7^{8,21}$.

Las limitaciones de nuestro estudio son varias. Este es un estudio descriptivo, no diseñado para 
evaluar el efecto del tratamiento en la sobrevida dado que las pacientes no fueron tratadas y seguidas de acuerdo a un protocolo pre-establecido. La valoración de Ki67, que permitiría comparar estos resultados con la mayoría de los datos publicados ${ }^{9}$, no se tuvo en el presente análisis. Las causas de muerte son aquellas obtenidas de nuestra base de datos clínica y de datos del Registro Civil, pudiendo haber un sesgo en la asignación de causa de muerte.

Tampoco evaluamos el sitio anatómico de las metástasis, que difiere también según el subtipo, como ha sido reportado por otros autores ${ }^{21}$.

A pesar de ello, mostramos la factibilidad de aplicar este modelo y sus resultados iniciales. Mientras la comparación de SG y SEE de tumores Luminales y no Luminales logra mostrar una diferencia estadísticamente significativa, reflejando principalmente el valor favorable, ya conocido, de la expresión del RE; observamos también una tendencia, no significativa, de mejor SEE en tumores definidos como Luminales A por sobre los Luminales B (SEE de 55,4 meses para los primeros y de 32,3 meses para los últimos). Igual hallazgo fue descrito para tumores con diferente grado histológico (mejor sobrevida en tumores no GH1-2 versus GH3). Probablemente por el tamaño acotado de nuestra población se limita la obtención de más conclusiones en el análisis de subgrupos.

\section{Conclusiones}

En comparación a pacientes con CM en etapa localizada, las pacientes con CMM al inicio de la enfermedad se presentan más frecuentemente con tumores HER2 positivos y de alto grado histológico. La clasificación clínica y patológica en subtipos de CM, basada en patrones de IHC, separa tumores con distinto comportamiento en pacientes con CMM al debut, y parece ser una herramienta útil y simple en nuestro medio, para categorizar esta enfermedad, evaluar su pronóstico a largo plazo y su respuesta a terapias personalizadas.

\section{Referencias}

1. http://www.ine.cl. Estadísticas Vitales. 2009. Visitado el 12-05-2013.

2. Berry D, Cronin K, Plevritis SK, Fryback DG, Clarke L, Zelen $\mathrm{M}$, et al. Effect of screening and adjuvant therapy on mortality from breast cancer. N Engl J Med 2005; 353 (17): 1784-92.

3. Siegel R, Naishadham D, Jemal A. Cancer Statistics, 2012. CA Cancer J Clin 2012; 62 (1): 10-29.

4. Tevaarwerk AJ, Gray RJ, Schneider BP, Smith M Lou, Wagner LI, Fetting JH, et al. Survival in patients with metastatic recurrent breast cancer after adjuvant chemotherapy: little evidence of improvement over the past 30 years. Cancer. 2013; 119 (6): 1140-8.

5. Perou CM, Sørlie T, Eisen MB, van de Rijn M, Jeffrey SS, Rees CA, et al. Molecular portraits of human breast tumours. Nature 2000; 406 (6797): 747-52.

6. Dowsett M, Sestak I, López-Knowles E, Sidhu K, Dunbier AK, Cowens JW, et al. Comparison of PAM50 Risk of Recurrence Score With Oncotype DX and IHC4 for Predicting Risk of Distant Recurrence After Endocrine Therapy. J Clin Oncol 2013; 31 (22): 2783-90.

7. Elston CW, Ellis IO. Pathological prognostic factors in breast cancer. I. The value of histological grade in breast cancer: experience from a large study with long-term follow up. Histopathology 1991; 19: 403-10.

8. Goldhirsch A, Wood WC, Coates AS, Gelber RD, Thürlimann B, Senn H-J. Strategies for subtypes-dealing with the diversity of breast cancer: highlights of the St. Gallen International Expert Consensus on the Primary Therapy of Early Breast Cancer 2011. Ann Oncol 2011; 22 (8): 1736-47.

9. Urruticoechea A, Smith IE, Dowsett M. Proliferation marker Ki-67 in early breast cancer. J Clin Oncol 2005; 23 (28): 7212-20.

10. Dawood S, Broglio K, Ensor J, Hortobagyi GN, Giordano SH. Survival differences among women with de novo stage IV and relapsed breast cancer. Ann Oncol 2010; 21 (11): 2169-74.

11. Walters S, Maringe C, Butler J, Rachet B, Barrett-Lee P, Bergh J, et al. Breast cancer survival and stage at diagnosis in Australia, Canada, Denmark, Norway, Sweden and the UK, 2000-2007: a population-based study. Br J Cancer 2013; 108 (5): 1195-208.

12. Paik S, Shak S, Tang G, Kim C, Baker J, Cronin M, et al. A multigene assay to predict recurrence of tamoxifentreated, node-negative breast cancer. N Engl J Med 2004; 351 (27): 2817-26.

13. Parker JS, Mullins M, Cheang MCU, Leung S, Voduc D, Vickery T, et al. Supervised risk predictor of breast cancer based on intrinsic subtypes. J Clin Oncol 2009; 27 (8): 1160-7.

14. Ma CX, Sánchez CG, Ellis MJ. Predicting endocrine therapy responsiveness in breast cancer. Oncology 2009; 23 (2): 133-42.

15. Drukker CA, Bueno-de-Mesquita JM, Retèl VP, van 
Harten WH, van Tinteren H, Wesseling J, et al. A prospective evaluation of a breast cancer prognosis signature in the observational RASTER study. Int J Cancer 2013; 133 (4): 929-36.

16. Drukker CA, van Tinteren H, Schmidt MK, Rutgers EJ, Bernards R, van de Vijver MJ, et al. Long-term impact of the 70-gene signature on breast cancer outcome. Breast Cancer Res Treat 2014; 143 (3): 587-92.

17. Goldhirsch A, Winer EP, Coates AS, Gelber RD, PiccartGebhart M, Thürlimann B, et al. Personalizing the treatment of women with early breast cancer: highlights of the St Gallen International Expert Consensus on the Primary Therapy of Early Breast Cancer 2013. Ann Oncol 2013; 24 (9): 2206-23.

18. De Azambuja E, Cardoso F, de Castro G, Colozza M, Mano MS, Durbecq V, et al. Ki-67 as prognostic mar- ker in early breast cancer: a meta-analysis of published studies involving 12,155 patients. Br J Cancer 2007; 96 (10): 1504-13.

19. Simpson BJF, Gray R, Dressler LG, Cobau CD, Falkson CI, Gilchrist KW, et al. Prognostic Value of Histologic Grade and Proliferative Activity in Axillary Node-Positive Breast Cancer: Results from the Eastern Cooperative Oncology Group Companion Study, EST 4189. J Clin Oncol 2000; 18 (10): 2059-69.

20. Galea MH, Blamey RW, Elston CE, Ellis IO. The Nottingham Prognostic Index in primary breast cancer. Breast Cancer Research and Treatment 1992; 22 (3): 207-19.

21. Kennecke H, Yerushalmi R, Woods R, Cheang MCU, Voduc D, Speers CH, et al. Metastatic behavior of breast cancer subtypes. J Clin Oncol 2010; 28 (20): 3271-7. 